\title{
Military Leadership Job and Skill Requirements: An Annotated Bibliography
}

\author{
Melvin J. Kimmel and Richard H. Balzer
}

Personnel Utilization Technical Area

Manpower and Personnel Research Laboratory

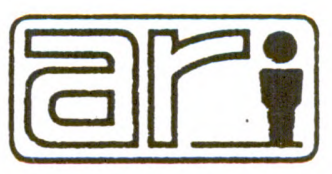

U. S. Army

Research Institute for the Behavioral and Social Sciences

June 1984 